\title{
Isometries in anti-de Sitter and conformal superspaces
}

\section{Piet Claus}

Instituut voor theoretische fysica, Katholieke Universiteit Leuven

B-3001 Leuven, Belgium

E-mail: piet.claus@fys.kuleuven.ac.bè

\section{Joachim Rahmfeld, Harlan Robins* and Jonathan Tannenhauser* \\ California Institute of Technology \\ Pasadena, CA 91125, USA, and \\ Caltech-USC Center for Theoretical Physics \\ University of Southern California, Los Angeles, CA 90089, USA \\ E-mail: rahmfeld@theory.caltech.edü, hrobins@theory.caltech.edú, \\ ijetannen@theory.caltech.edu'}

\section{Yonatan Zunger}

Physics Department, Stanford University

Stanford, CA 94305-4060, USA

E-mail: žunger@ieland.stanford.èü

ABSTRACT: We derive explicit forms for the superisometries of a wide class of supercoset manifolds, including those with fermionic generators in the stability group. We apply the results to construct the action of $\mathrm{SU}(2,2 \mid 4)$ on three supercoset manifolds: (10|32)-dimensional $A d S_{5} \times S^{5}$ superspace, (4|16)-dimensional conformal superspace, and a novel (10|16)-dimensional conformal superspace. Using superembedding techniques, we show, to lowest non-trivial order in the fermions, that at the boundary of $A d S_{5}$, the superisometries of the $A d S_{5} \times S^{5}$ superspace reduce to the standard $\mathcal{N}=4$ superconformal transformations. In particular, half of the 32 fermionic coordinates decouple from the superisometries.

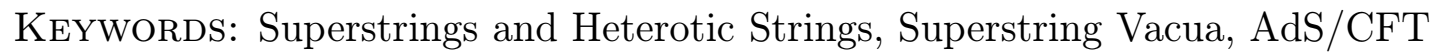
Coorrespondancét

*Department of Physics University of California at Berkeley, Berkeley, CA 94720, USA 


\section{Contents}

ii. Introduction

2. Superisometries of coset manifolds

3. Superisometries of $A d S_{5} \times S^{5}$ and conformal superspaces

'5.1.' Superisometries of $A d S_{5} \times S^{5}$ superspace

is.2 The $C_{(10 \mid 16)}$ superisometries

出. $A d S_{5} \times S^{5}$ superisometries and superconformal transformations

iA. The $\mathrm{SU}(2,2 \mid 4)$ algebra and spinors in $A d S_{5} \times S^{5}$ and conformal superspace

iĀ. $\overline{1}:$ The AdS decomposition

Ā $\overline{2}$ The superconformal decomposition

A

B.. The superisometries of $A d S_{5} \times S^{5}$ to quartic order in fermions

\section{Introduction}

A crucial element in the AdS/CFT correspondence iii is that the symmetry group $\mathrm{SU}(2,2 \mid 4)$ is shared by string theory in $A d S_{5} \times S^{5}$ and $\mathcal{N}=4$ super-Yang-Mills theory in four dimensions. In the string theory context, $\mathrm{SU}(2,2 \mid 4)$ appears as the group of isometries of $C_{(10 \mid 32)}$, the $A d S_{5} \times S^{5}$ superspace in which the (Green-Schwarz) string propagates. In the dual conformal field theory, $\mathrm{SU}(2,2 \mid 4)$ is the group of superconformal symmetries. Equivalently, we may take the gauge theory to be defined on a (4|16)-dimensional conformal superspace, denoted $C_{(4 \mid 16)}$, whose superisometries the superconformal transformations - again form the group $\mathrm{SU}(2,2 \mid 4)$. Both $C_{(10 \mid 32)}$ and $C_{(4 \mid 16)}$ are supercoset manifolds of $\mathrm{SU}(2,2 \mid 4)$, and the realization of $\mathrm{SU}(2,2 \mid 4)$ on the coordinates of these spaces is well studied.

The AdS/CFT correspondence identifies the boundary of anti-de Sitter space with the flat space on which the field theory is defined. If we are to implement the correspondence on the superspaces $C_{(10 \mid 32)}$ and $C_{(4 \mid 16)}$, then the isometries of $C_{(10 \mid 32)}$ 
must reduce, at the boundary of $A d S_{5}$, to the superconformal transformations of $C_{(4 \mid 16)}$. In particular, six bosonic and 16 fermionic coordinates must decouple from the $C_{(10 \mid 32)}$ superisometries in the boundary limit. The decoupling of the six bosons is familiar from the bosonic truncation of the $\mathrm{AdS} / \mathrm{CFT}$ correspondence. What is new is the decoupling of the fermions. The purpose of this paper is to show, to leading non-trivial order in an expansion in the fermionic coordinates, that the 32 fermions of the $A d S_{5} \times S^{5}$ superspace $C_{(10 \mid 32)}$ split into sets of 16 : one set may be identified, at the boundary of $A d S_{5}$, with the fermionic coordinates of $C_{(4 \mid 16)}$, and the other set decouples from the boundary superisometries.

Our first step is to derive explicit forms for the superisometries of these superspaces. In section 2,1, we develop general machinery for computing supercoset

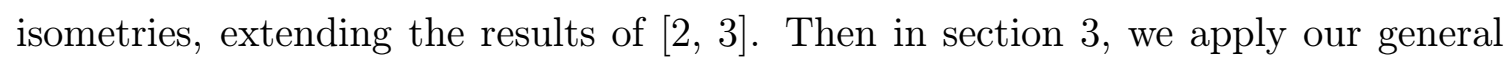
formulae to the supercosets $C_{(10 \mid 32)}$ and $C_{(4 \mid 16)}$, and also to a novel conformal superspace, denoted $C_{(10 \mid 16)}$, which is an enhancement of $C_{(4 \mid 16)}$ by six extra bosonic coordinates. With our choice of supercoset representatives, the boundary decoupling of the 16 fermionic coordinates from the $C_{(10 \mid 32)}$ superisometries is not obvious. We exhibit the decoupling in section 'i, by means of the superembedding approach

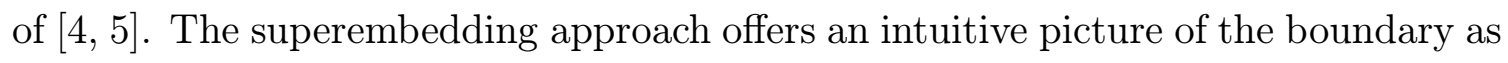
a (4|16)-dimensional brane embedded in $C_{(10 \mid 32)}$. The geometry of the embedding is captured in a certain matrix (the superembedding matrix), and the dynamics of the brane subjects this matrix to a constraint (the superembedding equation), whose solution provides us with the desired decomposition of bulk fermions. We find that the $C_{(10 \mid 32)}$ superisometries indeed reduce at the boundary to the $C_{(4 \mid 16)}$ superconformal transformations, with six bosonic and 16 fermionic coordinates decoupling.

The identification of bulk and boundary coordinates has many potential applications. One example is the study of Wilson loops. The AdS/CFT correspondence relates the expectation value $\langle W(\mathcal{C})\rangle$ of a Wilson loop operator along a contour $\mathcal{C}$ in the boundary $\mathcal{N}=4$ field theory to the action of the minimal-surface bulk string worldsheet ending on the loop $\mathcal{C}$. This aspect of the correspondence has been inves-

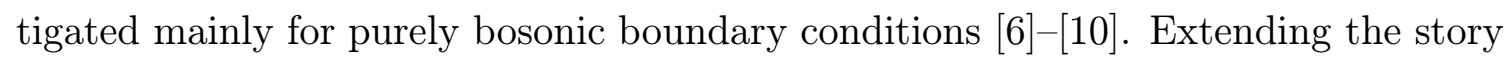
to the fully supersymmetric situation requires a detailed understanding of the relationship between bulk and boundary coordinates in superspace. The present work is a step in this direction, and a full discussion will be presented in [1]1]. The $C_{(10 \mid 16)}$ superspace plays an important role: the additional six bosonic coordinates are essentially identified with the loop variables of [i] 10$]$, which appear in the definition of the Wilson loop.

Another possible application is to the relation between gauged supergravity and

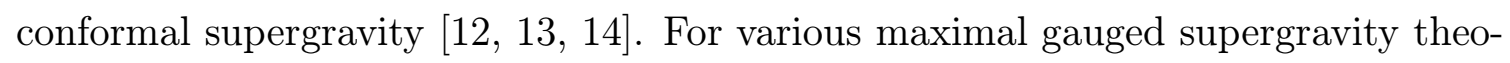
ries with AdS vacua, it has been shown that the field transformation rules reduce on the boundary of the AdS space to the transformation rules of conformal supergravity in one lower dimension [1] 
implemented as an identification of the local superspace translations. The present work identifies the rigid limit of these superspace translations in the case of fivedimensional gauged $\mathcal{N}=8$ supergravity (in the $A d S_{5}$ vacuum) and four-dimensional $\mathcal{N}=4$ conformal supergravity.

\section{Superisometries of coset manifolds}

Our goal is to compare the isometries of $A d S_{5} \times S^{5}$ superspace and conformal superspace, both of which are supercoset manifolds of the supergroup $\mathrm{SU}(2,2 \mid 4)$. In this section, we review a general scheme for describing supercoset manifolds and their isometries [2, 1030 , and obtain explicit expressions for the isometries of a class of coset supermanifolds that includes both superspaces.

Let $\mathbb{C}=\mathbb{G} / \mathbb{H}$ be a coset manifold, with coordinates $Z^{M}=\left(X^{\mu}, \theta^{\dot{\alpha}}\right)$, and let $\mathcal{G}(Z) \in \mathbb{C}$ be a coset representative. Given $g \in \mathbb{G}$, we can always find an element $h(g, Z)$ of the stability group $\mathbb{H}$ such that

$$
\mathcal{G}(Z) \longrightarrow g \mathcal{G}(Z) h^{-1}
$$

is an isometry of $\mathbb{C}$. The meaning of this equation is as follows. The element $g \in \mathbb{G}$ defines an isometry of the group $\mathbb{G}$ by left-multiplication. However, in general this transformation is not an isometry of $\mathbb{C}$ : if $\mathcal{G} \in \mathbb{C}, g \mathcal{G}$ need not be an element of $\mathbb{C}$. In fact,

$$
g \mathcal{G}(Z)=\mathcal{G}\left(Z^{\prime}\right) h
$$

for some $Z^{\prime}$ and some $h \in \mathbb{H}$, with $h$ depending in general on $g$ and $Z$. To get back to $\mathbb{C}$, we must compensate the transformation $g$ by right-multiplication by $h$.

Our characterization of the supercoset isometries will involve the Cartan 1-forms

$$
L(Z)=\mathcal{G}(Z)^{-1} d \mathcal{G}(Z),
$$

valued in the Lie algebra of $\mathbb{G}$. The Cartan forms trivially satisfy the Maurer-Cartan equation

$$
d L-L \wedge L=0
$$

They are left-invariant in the sense that they are unchanged under left-multiplication of $\mathcal{G}(Z)$ by an element $g \in \mathbb{G}$,

$$
(g \mathcal{G}(Z))^{-1} d(g \mathcal{G}(Z))-\mathcal{G}(Z)^{-1} d \mathcal{G}(Z)=0
$$

Using $\left({ }^{2}, \overline{2}^{\prime}\right)$, we may rewrite this as

$$
h^{-1} L\left(Z^{\prime}\right) h+h^{-1} d h-L(Z)=0 .
$$




\begin{tabular}{|c|c|c|c|}
\hline space & generators & bosonic generators & fermionic generators \\
\hline \hline $\mathbb{G}$ & $T_{\bar{A}}$ & $T_{\bar{a}}$ & $T_{\bar{\alpha}}$ \\
$\mathbb{C}$ & $C_{A}$ & $C_{a}$ & $C_{\alpha}$ \\
$\mathbb{H}$ & $H_{A^{\prime}}$ & $H_{a^{\prime}}$ & $H_{\alpha^{\prime}}$ \\
\hline
\end{tabular}

Table 1: Conventions for the generators of $\mathbb{G}, \mathbb{C}$, and $\mathbb{H}$.

Now let $g$ be an infinitesimal isometry. Let the associated coordinate transformation on the coset manifold ${ }^{1}$ be

$$
-\Xi^{M}=Z^{M}-Z^{M}
$$

and let the infinitesimal compensating transformation be

$$
h=1+\Lambda \text {. }
$$

Then the statement $\left(2 . \overline{6}^{\prime}\right)$ of the left-invariance of $L$ becomes

$$
\mathcal{L}_{\Xi} L+d \Lambda+[L(Z), \Lambda]=0
$$

where $\mathcal{L}_{\Xi}$ is the Lie derivative in the direction $\Xi$.

This is a $G$-covariant statement, but the form of (12.10 is not $G$-covariant: $L$ can take values in the full Lie superalgebra of $\mathbb{G}$, but $\Lambda$ is restricted to lie in the Lie superalgebra of $\mathbb{H}$. To write $\left(2 \overline{2} . \overline{1} 0_{1}^{\prime}\right)$ in a $G$-covariant form, we define the quantity $\Sigma=\Sigma^{\bar{A}} T_{\bar{A}}$ by

$$
\Sigma^{A^{\prime}}=\Lambda^{A^{\prime}}+\Xi^{M} L_{M}^{A^{\prime}}, \quad \Sigma^{A}=\Xi^{M} L_{M}^{A} .
$$

The notation is as in table i $1 \overline{1}$ : $\bar{A}$ runs over all the generators of $\mathbb{G}, A^{\prime}$ indexes the generators $H_{A^{\prime}}$ of the stability group $\mathbb{H}, A$ indexes the coset generators $C_{A}$ (i.e. the generators of $\mathbb{G}$ which are not generators of $\mathbb{H}$ ), and $L=L^{\bar{A}} T_{\bar{A}}=L_{M}^{\bar{A}} d Z^{M} T_{\bar{A}}$. Using the Maurer-Cartan equations, we can rephrase ( $\left.12 . \overline{1} 0^{\prime}\right)$ in the $G$-covariant form

$$
d \Sigma+[L, \Sigma]=0
$$

which is just the Killing equation for $\Sigma$.

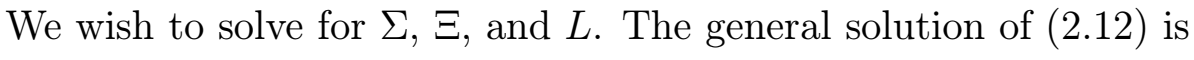

$$
\Sigma(Z)=\mathcal{G}(Z)^{-1} \Upsilon \mathcal{G}(Z),
$$

\footnotetext{
${ }^{1}$ For semi-simple groups $\mathbb{G}$ and $\mathbb{H}$, an isometry is equivalently characterized by the invariance of the line element

$$
g_{M N} d Z^{M} d Z^{N}=G_{A B} E_{M}^{A} E_{N}^{B} d Z^{M} d Z^{N},
$$

where $G_{A B}$ is the restriction to $\mathbb{C}$ of the Cartan-Killing metric on $\mathbb{G}$, and $E$ is the vielbein of the coset manifold. However, if $\mathbb{G}$ or $\mathbb{H}$ is not semi-simple - as is the case for Minkowski superspace and for the conformal superspaces we shall encounter in section - the Cartan-Killing metric is degenerate, and (2. $(2 . \bar{T})$ is not well defined. In this case, $g_{M N}$ must be replaced by an appropriate $\mathbb{G}$-invariant symmetric bilinear form, along the lines of [i] $\overline{1} \overline{7}]$.
} 
where $\Upsilon$ is a constant element of the Lie superalgebra of $\mathbb{G}$. To make further progress, we must choose a parametrization of the coset representative $\mathcal{G}(Z)$. We make the ansatz

$$
\mathcal{G}(Z)=v(X) e^{\Theta}
$$

where $v(X)$ is purely bosonic, $\Theta \equiv \Theta^{\alpha} C_{\alpha}$, and $\Theta^{\alpha}$ is related to the superspace fermionic coordinates $\theta^{\dot{\alpha}}$ by

$$
\Theta^{\alpha}=\theta^{\dot{\beta}} t_{\dot{\beta}}^{\alpha}(X) \text {. }
$$

We have the freedom to choose $t_{\dot{\beta}}^{\alpha}(X)$ arbitrarily. This freedom amounts to a gauge choice in superspace. For now, we will leave $t_{\dot{\beta}}{ }^{\alpha}(X)$ unspecified, and state our results for the isometries and the Cartan form in full generality, but as we shall see in section is, our formulae simplify drastically with an astute choice of superspace gauge.

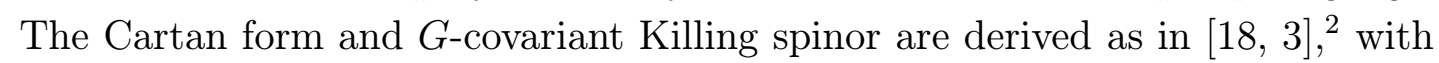
the result

$$
\begin{aligned}
L^{\bar{\alpha}} & =((D \Theta) A B)^{\bar{\alpha}}, \quad L^{\bar{a}}=L_{0}^{\bar{a}}-((D \Theta) A)^{\bar{\beta}} \Theta^{\alpha} f_{\alpha \bar{\beta}}^{\bar{a}}, \\
\Sigma^{\bar{\alpha}} & =((\mathcal{B} \Theta) A B)^{\bar{\alpha}}+\left(\Sigma_{0} \mathcal{M} \operatorname{coth} \mathcal{M} A B\right)^{\bar{\alpha}} \\
\Sigma^{\bar{a}} & =\Sigma_{0}^{\bar{a}}-\left(\Sigma_{0} A B\right)^{\bar{\beta}} \Theta^{\alpha} f_{\alpha \bar{\beta}}^{\bar{a}}-((\mathcal{B} \Theta) A)^{\bar{\beta}} \Theta^{\alpha} f_{\alpha \bar{\beta}}^{\bar{a}},
\end{aligned}
$$

where

$$
\begin{aligned}
A_{\bar{\alpha}}^{\bar{\beta}} & =2\left(\frac{\sinh ^{2} \mathcal{M} / 2}{\mathcal{M}^{2}}\right)_{\bar{\alpha}}^{\bar{\beta}} \quad, \quad B_{\bar{\alpha}}^{\bar{\beta}}=(\mathcal{M} \operatorname{coth} \mathcal{M} / 2)_{\bar{\alpha}}^{\bar{\beta}}, \\
(\mathcal{B} \Theta)^{\bar{\alpha}} & =\Theta^{\beta} \Sigma_{0}^{\bar{a}} f_{\bar{a} \beta}^{\bar{\alpha}}, \\
\mathcal{M}_{\bar{\alpha}}^{2 \bar{\beta}} & =f_{\bar{\alpha} \gamma}^{\bar{a}} \Theta^{\gamma} \Theta^{\delta} f_{\delta \bar{a}}^{\bar{\beta}}
\end{aligned}
$$

and the $f_{\bar{A}}^{\bar{C}} \bar{B}$ are structure constants of the isometry superalgebra. The bosonic components of $L$ and $\Sigma$ involve $L_{0}$ and $\Sigma_{0}$, the $\Theta=0$ values of the Cartan form and the Killing vector,

$$
L_{0}=v(X)^{-1} d v(X)=e^{a} C_{a}+\omega^{a^{\prime}} H_{a^{\prime}}, \quad \Sigma_{0}=v^{-1}(X) \Upsilon v(X)
$$

We have introduced the quantity

$$
(D \Theta)^{\bar{\alpha}} T_{\bar{\alpha}}=d \Theta^{\alpha} C_{\alpha}+d X^{\mu} e_{\mu}^{a} U_{a}^{\bar{\beta}} T_{\bar{\beta}}
$$

where the matrix $U_{a}^{\bar{\alpha}}$ depends on the superspace gauge choice $t_{\dot{\alpha}}{ }^{\beta}(X)$, and is given by

$$
U_{a}^{\bar{\alpha}} T_{\bar{\alpha}}=e_{a}{ }^{\mu}\left(\partial_{\mu} \Theta+\left[L_{0 \mu}, \Theta\right]\right)=e_{a}^{\mu}\left(\theta^{\dot{\alpha}} \partial_{\mu} t_{\dot{\alpha}}^{\alpha} C_{\alpha}+\theta^{\dot{\alpha}} t_{\dot{\alpha}}{ }^{\alpha} L_{0 \mu}^{\bar{a}} f_{\bar{a} \alpha}^{\bar{\beta}} T_{\bar{\beta}}\right) .
$$

\footnotetext{
${ }^{2}$ Our problem is more general than the one in $\left[1 \overline{1} \overline{8}_{1}^{\prime}, \overline{3}\right]$, since we allow $\mathbb{H}$ to contain fermionic generators. However, the methods used there to derive the Cartan forms and isometries are still valid.
} 
The last step in our program is the calculation of the superisometries $\Xi$. The Cartan form $L$ may be written as a linear combination of stability group generators and coset generators,

$$
L=L^{\bar{A}} T_{\bar{A}}=E^{A} C_{A}+\Omega^{A^{\prime}} H_{A^{\prime}} .
$$

The 1-form coefficients $E^{A}=E_{M}^{A} d Z^{M}$ are the vielbeins of the coset manifold, while the $\Omega^{A^{\prime}}$ make up the $\mathbb{H}$-connection, which is the analogue for coset manifolds of the usual spin connection. Inverting ( $\left(\overline{2} . \overline{1} \overline{1}_{1}^{\prime}\right)$, we obtain

$$
\Xi^{M}=\Sigma^{A}\left(E^{-1}\right)_{A}^{M} .
$$

We calculated $\Sigma^{A}$ in $\left(\overline{2}_{2} \cdot \overline{1} \overline{7}_{1}\right)$, but for $\left(\overline{2} . \overline{2} 3^{\prime}\right)$ to be truly useful, we need an expression for the inverse vielbein coefficients. We can compute the vielbein itself relatively straightforwardly, by comparing coefficients of the coset generators in $(\overline{2} . \overline{2} 2 \overline{2})$ and $\left(2 . \overline{1} \overline{6}_{1}\right)$. The result is

$$
E_{M}^{A}=\left(\begin{array}{cc}
e_{\mu}{ }^{b}(X) & 0 \\
0 & t_{\dot{\alpha}}^{\beta}(X)
\end{array}\right)\left(\begin{array}{cc}
\delta_{b}{ }^{a}+(U A \mathcal{Y})_{b}{ }^{a} & (U A B)_{b}{ }^{\alpha} \\
(A \mathcal{Y})_{\beta}{ }^{a} & (A B)_{\beta}{ }^{\alpha}
\end{array}\right),
$$

with

$$
\mathcal{Y}_{\bar{\alpha}}{ }^{a}=-\Theta^{\beta} f_{\beta \bar{\alpha}}{ }^{a} .
$$

If the fermionic generators all lie in $\mathbb{C}$, then

$$
\left(E^{-1}\right)_{A}^{M}=\left(\begin{array}{cc}
\delta_{a}{ }^{b} & -U_{a}{ }^{\beta} \\
-\left(B^{-1} \mathcal{Y}\right)_{\alpha}{ }^{b} & \left(B^{-1} \mathcal{Y} U\right)_{\alpha}{ }^{\beta}+(A B)^{-1}{ }_{\alpha}{ }^{\beta}
\end{array}\right)\left(\begin{array}{cc}
e_{b}{ }^{\mu} & 0 \\
0 & t_{\beta^{\alpha}}{ }^{\alpha}
\end{array}\right)
$$

and the superisometries are given by

$$
\begin{aligned}
\Xi^{\mu}= & \xi^{\mu}+\Sigma_{0}^{\alpha}\left(\mathcal{M}^{-1} \tanh \mathcal{M} / 2 \mathcal{Y}\right)_{\alpha}{ }^{a} e_{a}{ }^{\mu} \\
\Xi^{\dot{\beta}} t_{\dot{\beta}}{ }^{\alpha}= & \left(\Theta^{\beta} \Sigma_{0}^{\bar{a}} f_{\bar{a} \beta}^{\alpha}-\xi^{a} U_{a}{ }^{\alpha}\right)+\Sigma_{0}^{\beta}(\mathcal{M} \operatorname{coth} \mathcal{M})_{\beta}{ }^{\alpha}- \\
& -\Sigma_{0}^{\gamma}\left(\mathcal{M}^{-1} \tanh \mathcal{M} / 2\right)_{\gamma}{ }^{\beta}(\mathcal{Y} U)_{\beta}{ }^{\alpha} .
\end{aligned}
$$

All of the coset superspaces that arise as near-horizon limits of the standard superbranes $(M 2, M 5, D 3, D 1-D 5)$, including $A d S_{5} \times S^{5}$ superspace, are of this type.

Inverting $E$ is more difficult if the stability group contains fermionic generators. In this case, the matrices $A$ and $B$ appearing in ( $\left(2 . \overline{2} \overline{4}_{1}^{1}\right)$ are not square, so their inverses, which are needed in ( $\left.2.2 \overline{6}_{i}\right)$, are not well defined. However, if the (anti)commutator of every fermionic coset generator with every coset generator is itself in the coset,

$$
\left[C_{\alpha}, C_{A}\right\} \in \mathbb{C},
$$

then we may still use ( $\left(\overline{2} . \overline{2} \bar{z}_{1}\right)$ to calculate the superisometries, if we set the coordinates conjugate to the fermionic stability group generators to zero by hand. Both of the conformal superspaces we will study in section

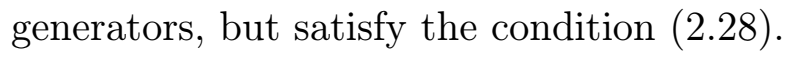




\begin{tabular}{|c|c|l|}
\hline \hline Operator & Weight & Name \\
\hline$P_{m}$ & 1 & Conformal Translations \\
\hline$Q$ & $1 / 2$ & Global Supersymmetries \\
\hline$M_{m n}$ & 0 & Lorentz Rotations \\
\hline$D$ & 0 & Dilatation \\
\hline$U_{j}{ }^{i}=\left(M_{m^{\prime} n^{\prime}}^{\prime}, P_{m^{\prime}}^{\prime}\right)$ & 0 & SO $(6)$ Rotations of $S^{5}$ \\
\hline$S$ & $-1 / 2$ & Special Supersymmetries \\
\hline$K_{m}$ & -1 & Special Conformal Transformations \\
\hline
\end{tabular}

Table 2: $\mathrm{SU}(2,2 \mid 4)$ generators in the superconformal basis. The $\mathrm{SO}(6)$ rotation $U$ is a linear combination of the $\mathrm{SO}(5)$ rotation $M_{m^{\prime} n^{\prime}}^{\prime}$ and the translation $P_{m^{\prime}}^{\prime}\left(m^{\prime}, n^{\prime}=1, \ldots, 5\right)$ on the 5 -sphere.

\section{Superisometries of $A d S_{5} \times S^{5}$ and conformal superspaces}

We now apply the formalism of the last section to compute the superisometries of three distinct cosets of $G=\mathrm{SU}(2,2 \mid 4) .{ }^{3}$ The generators of $\mathrm{SU}(2,2 \mid 4)$, together with their weights under dilatations, are listed in table 2

As noted in the introduction, the three coset spaces we study are:

(1) the $A d S_{5} \times S^{5}$ superspace

$$
C_{(10 \mid 32)}=\frac{\mathrm{SU}(2,2 \mid 4)}{\mathrm{SO}(1,4) \times \mathrm{SO}(5)},
$$

in which the Green-Schwarz string in an anti-de Sitter background naturally propagates;

(2) the conformal superspace

$$
C_{(4 \mid 16)}=\frac{\mathrm{SU}(2,2 \mid 4)}{\operatorname{Span}\left(\operatorname{iso}(1,3)_{K} \oplus D \oplus \operatorname{so}(6) \oplus S\right)},
$$

on which the dual $\mathcal{N}=4$ super-Yang-Mills theory may be formulated; and

(3) a novel conformal superspace

$$
C_{(10 \mid 16)}=\frac{\mathrm{SU}(2,2 \mid 4)}{\operatorname{Span}\left(\operatorname{iso}(1,3)_{K} \oplus \operatorname{so}(5) \oplus S\right)},
$$

which differs from $C_{(4 \mid 16)}$ by the addition of six bosonic coordinates. This superspace will find application in the study of supersymmetric Wilson loops ii i i .

The division of the $\mathrm{SU}(2,2 \mid 4)$ generators into coset and stability group generators for each coset space is shown in table

\footnotetext{
${ }^{3}$ Our conventions for spinors and for the $\mathrm{SU}(2,2 \mid 4)$ algebra are given in appendix A.
} 


\begin{tabular}{|c||c|c|c|}
\hline & $C_{(10 \mid 32)}$ & $C_{(10 \mid 16)}$ & $C_{(4 \mid 16)}$ \\
\hline \hline $\mathbb{C}$ & $\frac{1}{2}\left(P_{m}+K_{m}\right), P_{m^{\prime}}^{\prime}, D, Q, S$ & $P_{m}, P_{m^{\prime}}^{\prime}, D, Q$ & $P_{m}, Q$ \\
\hline $\mathbb{H}$ & $\frac{1}{2}\left(P_{m}-K_{m}\right), M_{m n}, M_{m^{\prime} n^{\prime}}^{\prime}$ & $K_{m}, M_{m n}, M_{m^{\prime} n^{\prime}}^{\prime}, S$ & $K_{m}, D, P_{m^{\prime}}^{\prime}, M_{m n}, M_{m^{\prime} n^{\prime}}^{\prime}, S$ \\
\hline
\end{tabular}

Table 3: Coset decompositions of SU $(2,2 \mid 4)$ Generators

\subsection{Superisometries of $A d S_{5} \times S^{5}$ superspace}

Super-horospheric coordinates. We begin by describing our choice of coordinates on the $A d S_{5} \times S^{5}$ superspace $C_{(10 \mid 32)}$. For bosonic coordinates on $A d S_{5}$ we choose the horospheric coordinates $\left(x^{m}, \rho\right)$, which appear naturally in the nearhorizon limit of brane solutions in supergravity. The $x^{m}$ are four Cartesian bulk coordinates parallel to the brane, while $\rho$ is the bulk coordinate perpendicular to the brane. In these coordinates the $A d S_{5}$ metric has the form

$$
d s^{2}=\rho^{2} d x^{2}+\left(\frac{R}{\rho}\right)^{2} d \rho^{2}
$$

where $R$ is the characteristic length ("radius") of $A d S_{5}$. In the following we will set $R=1$. The boundary of $A d S_{5}$ is located at $\rho=\infty$. The coordinates on $S^{5}$ are denoted by $\phi^{m^{\prime}}$. Sometimes we group the coordinates of the sphere and the radial coordinate of $A d S_{5}$ into a system of 6 Cartesian coordinates $y^{I}$, with

$$
\rho=|y|
$$

We wish to extend these bosonic coordinates to a coordinate system on $C_{(10 \mid 32)}$ in which the superisometries take as simple a form as possible. This involves fixing the arbitrary matrix $t_{\dot{\beta}}{ }^{\alpha}$ in $(\overline{2} \cdot 15)$. To guide our choice, we notice that all fermionic dependence in (12.16 of $t_{\dot{\beta}}{ }^{\alpha}(X)$ would simultaneously simplify $D \Theta$ and $\Theta$, and so render the calculation of the superisometries tractable.

The most obvious choice $t_{\dot{\beta}}{ }^{\alpha}(X)=\delta_{\dot{\beta}}{ }^{\alpha}$ (Wess-Zumino gauge) simplifies $\Theta$, but $D \Theta$ remains complicated. Another option is to choose $t_{\dot{\beta}}{ }^{\alpha}$ such that, if $\epsilon^{\dot{\alpha}}$ is a constant spinor, the spinor

$$
\epsilon^{\alpha}(X)=\epsilon^{\dot{\beta}} t_{\dot{\beta}}^{\alpha}(X)
$$

satisfies the Killing equation

$$
\left(\delta_{\beta}^{\alpha} d+L^{\bar{a}} f_{\bar{a} \beta}^{\alpha}\right) \epsilon^{\beta}(X)=0 .
$$

This ensures that

$$
U_{a}^{\bar{\alpha}}=0 .
$$


In this gauge, called Killing gauge [i]

$$
(D \Theta)^{\alpha}=(d \theta)^{\dot{\beta}} t_{\dot{\beta}}{ }^{\alpha} \quad \text { and } \quad(D \Theta)^{\alpha^{\prime}}=0 .
$$

However, with this choice the expressions for the superisometries are not translationally invariant, and $\Theta$ itself is a rather messy function of $\theta$. We therefore work in a different gauge, which we now introduce.

Let us write the fermionic generators of $\mathrm{SU}(2,2 \mid 4)$ in the superconformal decomposition (for details see appendix A.2)

$$
\mathcal{Q}_{\alpha}{ }^{i}=\left(\begin{array}{c}
Q_{\alpha}{ }^{i} \\
S_{\alpha}{ }^{i}
\end{array}\right)
$$

For the fermionic parametrization of the coset we choose

$$
\begin{aligned}
\Theta & =\bar{\Theta}_{i} \mathcal{Q}^{i}+\overline{\mathcal{Q}}_{i} \Theta^{i} \\
& =\left(u^{-1}\right)_{i}{ }^{j} \bar{\theta}_{i} \rho^{1 / 2} Q^{i}+\left(u^{-1}\right)_{i}{ }^{j} \bar{\vartheta}_{j} \rho^{-1 / 2} S^{i}+\bar{Q}_{i} \rho^{1 / 2} \theta^{j} u_{j}{ }^{i}+\bar{S}_{i} \rho^{-1 / 2} \vartheta^{j} u_{j}{ }^{i}
\end{aligned}
$$

Here $u_{i}{ }^{j}=u_{i}{ }^{j}(\phi)$ is a coset representative of $\mathrm{SO}(6) / \mathrm{SO}(5)$; the indices $i, j$ are spinor indices of $\mathrm{SO}(6)$. We name the gauge defined by ( $\left(\overline{1}_{-} . \overline{1} \overline{1}_{1}^{\prime}\right)$ partial-Killing gauge, as it is intermediate between the Wess-Zumino and Killing gauges. In this gauge,

$$
U_{\rho}^{\bar{\alpha}}=U_{m^{\prime}}^{\bar{\alpha}}=0
$$

but $U_{\mu}^{\bar{\alpha}}$ need not equal zero; the Killing equation is satisfied in the $S^{5}$ and radial $A d S$ directions, but not in the directions parallel to the boundary. The coordinates $Z^{M}=\left\{x^{m}, \rho, \phi^{m^{\prime}}, \theta^{i}, \vartheta^{i}, \bar{\theta}_{i}, \bar{\vartheta}_{i}\right\}$ are called super-horospheric coordinates.

The $A d S_{5} \times S^{5}$ supergeometry and isometries. The supercoset $C_{(10 \mid 32)}$ is maximally supersymmetric; the stability group contains no fermionic generators. The isometries are calculated from $\left(\overline{2} . \overline{2} \overline{7}_{1}\right)$.

The geometry is given by the supervielbein

$$
E=E^{\bar{M}} C_{\bar{M}}=E^{m} P_{m}+E^{\rho} D+E^{m^{\prime}} P_{m^{\prime}}+\left(\bar{Q}_{i} E_{Q}^{i}+\text { h.c. }\right)+\left(\bar{S}_{i} E_{S}^{i}+\text { h.c. }\right),
$$

where

$$
\begin{aligned}
E^{m}=\rho & {\left[x^{n}\left(\delta_{n}{ }^{m}-\frac{1}{2}\left(\frac{1}{\rho}\right)^{2} \bar{\vartheta}_{i} \gamma_{n} \vartheta^{j} \bar{\vartheta}_{j} \gamma^{m} \vartheta^{i}\right)+\right.} \\
& +\left(\frac{1}{2} d \bar{\theta}_{i} \gamma^{m} \theta^{i}+\frac{1}{4} \bar{\theta}_{i} d \vartheta^{j} \bar{\theta}_{j} \gamma^{m} \theta^{i}+\text { h.c. }\right)+ \\
& \left.+\left(\frac{1}{\rho}\right)^{2}\left(\frac{1}{2} d \bar{\vartheta}_{i} \gamma^{m} \vartheta^{i}+\frac{1}{4} \bar{\vartheta}_{i} d \theta^{j} \bar{\vartheta}_{j} \gamma^{m} \vartheta^{i}+\text { h.c. }\right)\right]+\mathcal{O}(\theta \wedge \vartheta)
\end{aligned}
$$




$$
\begin{aligned}
E^{\rho} & =\frac{1}{\rho}\left[d \rho-\frac{1}{2}\left(d \bar{\theta}_{i} \vartheta^{i}-d \bar{\vartheta}_{i} \theta^{i}+\text { h.c. }\right) \rho\right]+\mathcal{O}(\theta \wedge \vartheta) \\
E^{m^{\prime}} & =e^{m^{\prime}}-i \frac{1}{2}\left(d \bar{\theta}_{i} \vartheta^{j}+d \bar{\vartheta}_{i} \theta^{j}+d x^{m} \bar{\vartheta}_{i} \gamma_{m} \vartheta^{j}-\text { h.c. }\right)\left(u \gamma^{\prime m^{\prime}} u^{-1}\right)_{j}{ }^{i}+\mathcal{O}(\theta \wedge \vartheta) \\
E_{Q}^{i} & =\rho^{1 / 2}\left[d \theta^{j}-d x^{m} \gamma_{m} \vartheta^{j}+\frac{1}{3} \theta^{k}\left(d \bar{\vartheta}_{k} \theta^{j}-\bar{\theta}_{k} d \vartheta^{j}\right)\right] u_{j}^{i}+\mathcal{O}(\theta \wedge \vartheta) \\
E_{S}^{i} & =\rho^{-1 / 2}\left[d \vartheta^{i}+\frac{1}{3} \vartheta^{k}\left(2 d \bar{\theta}_{k} \vartheta^{j}-\bar{\vartheta}_{k} d \theta^{j}\right)+d x^{m} \vartheta^{k} \bar{\vartheta}_{k} \gamma_{m} \vartheta^{j}\right] u_{j}^{i}+\mathcal{O}(\theta \wedge \vartheta) .(3.1)
\end{aligned}
$$

Here $\mathcal{O}(\theta \wedge \vartheta)$ stands for terms containing both $\theta^{i}$ and $\vartheta^{i}$. As we have noted, it is sometimes convenient to replace the coordinates $\left(\rho, \phi^{m^{\prime}}\right)$ by cartesian coordinates $y^{I}$. In these coordinates, $E^{\rho}$ and $E^{m^{\prime}}$ are subsumed into

$$
\begin{aligned}
E^{I}=\frac{1}{\rho}\left[d y^{I}-\frac{1}{2}\left(\left(d \bar{\theta}_{i} \vartheta^{j}+d x^{m} \bar{\vartheta}_{i} \gamma_{m} \vartheta^{j}\right)\left(\delta^{I J} \mathbb{1}-\hat{\gamma}^{\prime I J}\right)_{j}{ }^{i} y_{J}+\text { h.c. }\right)+\right. \\
\left.+\frac{1}{2}\left(d \bar{\vartheta}_{i} \theta^{j}\left(\delta^{I J} \mathbb{1}+\hat{\gamma}^{I J}\right)_{j}{ }^{i} y_{J}+\text { h.c. }\right)\right]+\mathcal{O}(\theta \wedge \vartheta)
\end{aligned}
$$

The superisometries then follow from substituting into $\left(\overline{2} . \overline{2} \overline{7}_{1}\right)$ :

$$
\begin{aligned}
\delta x^{m}= & -\xi_{C}^{m}(x)-\frac{1}{2}\left(\bar{\epsilon}_{i}(x) \gamma^{m} \theta^{i}+\text { h.c. }\right)-\frac{1}{4}\left(\bar{\eta}_{i} \theta^{j} \bar{\theta}_{j} \gamma^{m} \theta^{i}+\text { h.c. }\right)- \\
& -\left(\frac{R}{\rho}\right)^{2}\left[\Lambda_{K}^{m}+\frac{1}{2}\left(\bar{\eta}_{i} \gamma^{m} \vartheta^{i}+\text { h.c. }\right)+\frac{1}{4}\left(\bar{\epsilon}(x)_{i} \vartheta^{j} \bar{\vartheta}_{j} \gamma^{m} \vartheta^{i}+\text { h.c. }\right)\right]+ \\
& +\mathcal{O}(\theta \wedge \vartheta) \\
\delta \rho= & \Lambda_{D}(x) \rho+\frac{1}{2}\left(\bar{\epsilon}_{i}(x) \vartheta^{i}-\bar{\eta}_{i} \theta^{i}+\text { h.c. }\right) \rho+\mathcal{O}(\theta \wedge \vartheta) \\
\delta \phi^{m^{\prime}}= & -\xi^{m^{\prime}}(\phi)+\frac{i}{2}\left(\bar{\epsilon}_{i}(x) \vartheta^{j}+\bar{\eta}_{i} \theta^{j}-\text { h.c. }\right)\left(u \gamma^{\prime m^{\prime}} u^{-1}\right) e_{\bar{m}^{\prime}} m^{\prime}+\mathcal{O}(\theta \wedge \vartheta) \\
\delta y^{I}= & \Lambda_{D}(x) y^{I}-\Lambda_{\mathrm{SO}(6)}^{I J} y_{J}+\frac{1}{2}\left(\bar{\epsilon}_{i}(x) \vartheta^{j}\left(\delta^{I J} \mathbb{1}-\hat{\gamma}^{\prime I J}\right)_{j}{ }^{i} y_{J}+\text { h.c. }\right)- \\
& -\frac{1}{2}\left(\bar{\eta}_{i} \theta^{j}\left(\delta^{I J} \mathbb{1}+\hat{\gamma}^{\prime I J}\right)_{j}{ }^{i} y_{J}+\text { h.c. }\right)+\mathcal{O}(\theta \wedge \vartheta) \\
\delta \theta^{i}= & -\epsilon^{i}(x)-\frac{1}{2} \Lambda_{D}(x) \theta^{i}-\frac{1}{4} \Lambda_{M}(x) \cdot \gamma \theta^{i}-\frac{1}{4} \theta^{i} \Lambda_{\mathrm{SO}(6)}^{I J}\left(\hat{\gamma}_{I J}^{\prime}\right)_{j}{ }^{i}- \\
& -\left(\frac{R}{\rho}\right)^{2}\left[\Lambda_{K}^{m}+\frac{1}{2}\left(\bar{\eta}_{j} \gamma^{m} \vartheta^{j}+\text { h.c. }\right)+\frac{1}{4}\left(\bar{\epsilon}_{j}(x) \vartheta^{k} \bar{\vartheta}_{k} \gamma^{m} \vartheta^{j}+\text { h.c. }\right)\right] \gamma_{m} \vartheta^{i}- \\
& -\frac{2}{3} \theta^{j}\left(2 \bar{\eta}_{j} \theta^{i}-\bar{\theta}_{j} \eta^{i}\right)+\mathcal{O}(\theta \wedge \vartheta), \\
\delta \vartheta^{i}= & -\eta^{i}+X_{K} \theta^{i}+\frac{1}{2} \Lambda_{D}(x) \vartheta^{i}-\frac{1}{4} \Lambda_{M}(x) \cdot \gamma \vartheta^{i}-\frac{1}{4} \vartheta^{i} \Lambda_{\mathrm{SO}(6)}^{I J}\left(\hat{\gamma}_{I J}^{\prime}\right)_{j}{ }^{i}- \\
& -\frac{2}{3} \vartheta^{j}\left(2 \epsilon_{j}(x) \vartheta^{j}-\bar{\vartheta}_{j} \epsilon^{i}(x)\right)+\mathcal{O}(\theta \wedge \vartheta) .
\end{aligned}
$$


We have written these transformations in terms of the $x$-dependent parameters of superconformal transformations,

$$
\begin{aligned}
\xi_{C}^{m}(x) & =a^{m}+\lambda_{M}^{m n} x_{n}+\lambda_{D} x^{m}+\left(x^{2} \Lambda_{K}^{m}-2 x^{m} x \cdot \Lambda_{K}\right), \\
\Lambda_{M}^{m n}(x) & =\lambda_{M}^{m n}-4 x^{[m} \Lambda_{K}^{n]} \\
\Lambda_{D}(x) & =\lambda_{D}-2 x \cdot \Lambda_{K}, \\
\Lambda_{K}^{m} & =\Lambda_{K}^{m} \\
\epsilon^{i}(x) & =\left(\epsilon^{i}+x^{m} \gamma_{m} \eta^{i}\right) .
\end{aligned}
$$

Here $a^{m}, \lambda_{M}^{m n}, \lambda_{D}, \Lambda_{K}^{m}$ are the constant parameters of translations, Lorentz rotations, dilatations and special conformal transformations; $\epsilon$ and $\eta$ parametrize supersymmetries and special supersymmetries; and the $\Lambda_{\mathrm{SO}(6)}^{I J}$ are the parameters of the $\mathrm{SO}(6) R$-symmetry.

In the boundary limit $\rho \rightarrow \infty$, the radial coordinate $\rho$ decouples from the bosonic isometries $\delta x^{\mu}$, and these isometries reduce at the boundary to the conformal transformations of conformal space. We likewise expect that 16 fermions decouple from the superisometries in the boundary limit, and that the superisometries reduce in that limit to the superconformal transformations of conformal superspace. The decoupling of the fermions is not evident in (13.16), though. In fact, from (16) it would seem that the boundary isometries depend on all 32 fermionic coordinates. We will resolve this puzzle in section 零, by exhibiting a change of coordinates in which the decoupling of 16 fermions is apparent. To make the decoupling precise, we need the form of the superconformal transformations of the boundary. We now study these transformations in the context of the novel conformal superspace $C_{(10 \mid 16)}$ defined above.

\subsection{The $C_{(10 \mid 16)}$ superisometries}

The novel conformal superspace $C_{(10 \mid 16)}$ is an enlargement of the conformal Minkowski superspace $C_{(4 \mid 16)}$ by the six bosonic coordinates $v^{I}=\left(v, \varphi^{m^{\prime}}\right)$. At the level of the $\mathrm{SU}(2,2 \mid 4)$ algebra, this enlargement is realized by reclassifying the generator $D$ (corresponding to $\rho$ ) and the generators of $\mathrm{SO}(6) / \mathrm{SO}(5)$ (corresponding to the sphere coordinates) as coset generators, rather than as stability group generators. Fermionically, $C_{(10 \mid 16)}$ differs from that of $C_{(10 \mid 32)}$ in that generators $S$ are assigned to the stability group. The 16 fermionic coordinates are defined by

$$
\Theta=\left(u^{-1}\right)_{i}{ }^{j} \bar{\lambda}_{i} v^{1 / 2} Q^{i}+\bar{Q}_{i} v^{1 / 2} \lambda^{j} u_{j}^{i},
$$

which is the natural adaptation of $\left(\bar{n}_{3} . \overline{1} \overline{1}_{1}^{\prime}\right)$. Within this coset this choice corresponds to the Killing gauge.

The coordinates $z^{M}$ of $C_{(10 \mid 16)}$ conjugate to the generators $\left(P_{m}, Q^{i}, D, P_{m^{\prime}}^{\prime}\right)$ are $\left(w^{m}, \lambda_{i}^{\alpha}, v, \varphi^{m^{\prime}}\right)$. The supervielbein $e$

$$
e=e^{m} P_{m}-e^{v} D+e^{m^{\prime}} P_{m^{\prime}}+\left(\bar{Q}_{i} e^{i}+\text { h.c. }\right)
$$


of $C_{(10 \mid 16)}$ may be obtained either by direct calculation or by setting $\vartheta=d \vartheta=0$, $\left(x^{m}, y^{I}, \theta^{i}\right)=\left(w^{m}, v^{I}, \lambda^{i}\right)$ in the Cartan form of $A d S_{5} \times S^{5}$ superspace. The result is essentially the vielbein of flat superspace. The isometries are given by

$$
\begin{aligned}
\delta w^{m}= & -\xi_{C}^{m}(w)-\frac{1}{2}\left(\bar{\epsilon}_{i}(w) \gamma^{m} \lambda^{i}+\text { h.c. }\right)+\frac{1}{2}\left(\bar{\lambda}_{i} \eta^{j}-\bar{\eta}_{i} \lambda^{j}\right) \bar{\lambda}_{j} \gamma^{m} \lambda^{i}-\frac{1}{2} \bar{\lambda}_{i} X_{K} \lambda^{j} \bar{\lambda}_{j} \gamma^{m} \lambda^{i}, \\
\delta \lambda^{i}= & -\epsilon^{i}(w)-\frac{1}{2} \Lambda_{D}(w) \lambda^{i}-\frac{1}{4} \Lambda_{M} \cdot \gamma \lambda^{i}-\frac{1}{4} \lambda^{j} \Lambda_{\mathrm{SO}(6)}^{I J}\left(\hat{\gamma}_{I J}^{\prime}\right)_{j}{ }^{i}-\lambda^{j}\left(2 \bar{\eta}_{j} \lambda^{i}-\bar{\lambda}_{j} \eta^{i}\right)- \\
& -\frac{1}{2} \lambda^{j} \bar{\lambda}_{j} X_{K} \lambda^{i} \\
\delta v^{I}= & \Lambda_{D}(w) v^{I}-\Lambda_{\mathrm{SO}(6)}^{I J} v_{J}-\left(\left(\bar{\eta}_{i} \lambda^{j}+\frac{1}{2} \bar{\lambda}_{i} X_{K} \lambda^{j}\right)\left(\mathbb{1} \delta^{I J}+\hat{\gamma}^{I J}\right)_{j}{ }^{i} v_{J}+\text { h.c. }\right)
\end{aligned}
$$

These transformations are precisely the superconformal transformations derived in $[\underline{1} \overline{\underline{1}} \overline{9}]$ via supertwistors, extended to the bulk directions $v^{I}$. They realize the $\operatorname{SU}(2,2 \mid 4)$ algebra on the $10+16$ coordinates, as desired.

The $v^{I}$ coordinates are completely decoupled from the transformations of $x$ and $\theta$. Their associated generators can therefore be freely moved into the stability group, to give $C_{(4 \mid 16)}$, without affecting the isometries. Indeed, $\delta w^{m}$ and $\delta \lambda^{i}$ in $\left(\overline{3}_{2} . \overline{2} 0_{1}^{\prime}\right)$ are exactly the superisometries of conformal Minkowski superspace.

\section{4. $A d S_{5} \times S^{5}$ superisometries and superconformal transformations}

In the bosonic truncation of the AdS/CFT correspondence, the isometries of the bulk space restrict at the boundary to the conformal symmetries of the boundary theory. Any sensible extension of AdS/CFT to superspace must preserve this feature; namely, the superisometries of the bulk space must reduce in the boundary limit to the superconformal transformations of conformal superspace. In particular, in the example we studied in section 16 of the 32 fermionic coordinates of $C_{(10 \mid 32)}$ must decouple from the superisometries in the limit $\rho \rightarrow \infty$. But no decoupling of this sort is apparent in ( $\left(\bar{B}_{1}\right)$, nor is it clear what the proper relationship is between the $C_{(4 \mid 16)}$ fermionic coordinates $\lambda$ and the $C_{(10 \mid 32)}$ fermions $\theta$ and $\vartheta$.

We can state the difficulty another way. If the AdS/CFT correspondence extends to superspace, then it must be possible to carve out of the $10+32$ coordinates of $A d S_{5} \times S^{5}$ superspace a set of $4+16$ supercoordinates $z^{M}=\left(w^{m}, \lambda^{i}\right)$, with

$$
\begin{aligned}
w^{m} & =w^{m}\left(x^{m}, y^{I}, \theta, \vartheta\right), \\
\lambda & =\lambda\left(x^{m}, y^{I}, \theta, \vartheta\right),
\end{aligned}
$$

and with the property that

$$
\left.\delta_{(10 \mid 32)} z^{M}\right|_{\rho=\infty}=\delta_{(4 \mid 16)} z^{M}
$$


Here $\delta_{(10 \mid 32)}$ and $\delta_{(4 \mid 16)}$ denote the superisometries $\left(\bar{B}_{-1} \overline{1} \overline{6}_{1}^{\prime}\right)$ and the superconformal transformations $\left(\overline{3} .20^{\prime}\right)$. We are thus asking for a (4|16)-dimensional brane $C_{(4 \mid 16)}$ embedded in $C_{(10 \mid 32)}$ at $\rho=\infty$, on which the superisometries of $C_{(10 \mid 32)}$ restrict to the superisometries of conformal superspace. The equations (1, $\bar{L}_{2}$ ) are overconstrained, so the existence of a solution is non trivial.

Nonetheless, we will find one. Our method uses the superembedding formalism

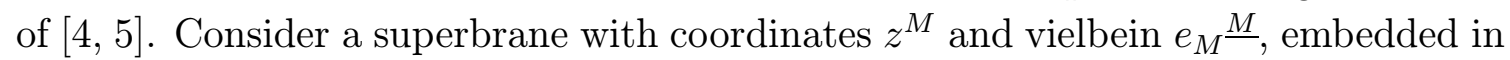
a larger target superspace with coordinates $Z^{\mathbf{M}}$ and vielbein $E_{\mathbf{M}} \underline{\mathbf{M}}$. ${ }^{4}$ The geometry of the embedding is encoded in the superembedding matrix

$$
\hat{E}_{\underline{M}}^{\underline{\underline{M}}} \equiv\left(e^{-1}\right)_{\underline{M}}^{M} \partial_{M} Z^{\mathbf{M}} E_{\mathbf{M}^{\underline{M}}} \text {. }
$$

The dynamics of the embedded superbrane are contained in the requirement that the superembedding matrix satisfy the superembedding equation

$$
\hat{E}_{\underline{\alpha}} \underline{\mu}=0
$$

where $\mu$ runs over the bosonic directions of the target space. The superembedding equation states that the fermionic tangent space of the superbrane lies entirely within the fermionic tangent space of the target space, and does not protrude into the bosonic part of the tangent space of the target.

For us, the embedded superbrane is the boundary space $C_{(4 \mid 16)}$, and the target space in which it is embedded is the bulk space $C_{(10 \mid 32)}$. The superembedding matrix is given in terms of the vielbein of $C_{(10 \mid 32)},{ }^{5}$ the unknown coordinate transformation ( $\left.\bar{A}_{-} \overline{1}_{-}\right)$, and the vielbein of $C_{(4 \mid 16)}$, which is just the vielbein of flat superspace,

$$
\begin{aligned}
e^{\underline{\underline{m}}} & =d w^{m} \delta \frac{\underline{m}}{m}+\frac{1}{2} d \bar{\lambda}_{i} \gamma^{\underline{m}} \lambda^{i}-\frac{1}{2} \bar{\lambda}_{i} \gamma^{\underline{m}} \lambda^{i}, \\
e^{\underline{\underline{i}}} & =d \lambda^{\underline{i}}
\end{aligned}
$$

Thus the superembedding equation constrains the coordinate transformation ( $\left.\bar{A}_{-} \bar{L}_{1}^{\prime}\right)$. The most naive identifications $w^{m}=x^{m}, \lambda^{i}=\theta^{i}$ violate ('A.4'). However, the corrected transformations

$$
\begin{aligned}
w^{m} & =x^{m}-\frac{1}{4}\left(\bar{\theta}_{i} \vartheta^{j}-\bar{\vartheta}_{i} \theta^{j}\right)\left(\bar{\theta}_{j} \gamma^{\mu} \theta^{i}\right), \\
\lambda_{\alpha}{ }^{i} & =\theta_{\alpha}{ }^{i}+\frac{1}{3} \theta_{\alpha}{ }^{j}\left(2 \bar{\vartheta}_{j} \theta^{i}-\bar{\theta}_{j} \vartheta^{i}\right)
\end{aligned}
$$

\footnotetext{
${ }^{4}$ Underlined and non-underlined indices refer to local Lorentz and general coordinate frames, respectively.

${ }^{5}$ Extracting non-trivial information from the superembedding matrix requires more terms in the expansion of the vielbein of $C_{(10 \mid 32)}$ than were presented in section $\overline{3} \cdot$. In appendix B we give the bulk vielbein and superisometries to quartic order in the fermions, in the relevant limit $\rho \rightarrow \infty$.
} 
solve (14.4. be developed to all orders $\theta$ and $\vartheta .{ }^{6}$ Moreover, the coordinate transformations ( $\left(1 \overline{4} .{ }^{4}\right)$ satisfy $\left(\bar{A}_{-} \overline{2}^{\prime}\right): 16$ fermionic degrees of freedom do decouple from the $C_{(10 \mid 32)}$ superisometries in the limit $\rho \rightarrow \infty$, and the superisometries indeed reduce in that limit to the superisometries of conformal superspace.

The redefinitions $\left({ }^{\prime} . \overline{6}_{-}^{*}\right)$ can be extended to a map from $C_{(10 \mid 32)}$ to the novel conformal superspace $C_{(10 \mid 16)}$, with coordinates $\left(w^{m}, v^{I}, \lambda^{i}\right)$, by supplementing (14.6. with the transformations

$$
v^{I}=y^{I}-\frac{1}{2} \bar{\vartheta}_{i} \theta^{j}\left(\delta^{I J} \mathbb{1}-\hat{\gamma}^{I J}\right)_{j}{ }^{i} y_{J}-\frac{1}{2} \bar{\theta}_{j} \vartheta^{i}\left(\delta^{I J} \mathbb{1}+\hat{\gamma}^{I J}\right)_{i}{ }^{j} y_{J}
$$

Again, the superisometries of the bulk restrict at the boundary to the superconformal transformations of the novel conformal superspace, in the sense of ('A.

\section{Acknowledgments}

We have enjoyed useful discussions with Djordje Minic, Hirosi Ooguri and Dmitri Sorokin. We are particularly thankful to Renata Kallosh for comments and extensive collaboration during many stages of this work. J.R. was supported by the Caltech Discovery Fund and DE-FG03-92-ER40701. H.R. and J.T. are supported in part by NSF grant PHY-95-14797 and DOE grant DE-AC03-76SF00098. Y.Z. was partially supported by an NSF graduate research fellowship.

\section{A. The $\mathrm{SU}(2,2 \mid 4)$ algebra and spinors in $A d S_{5} \times S^{5}$ and conformal superspace}

The $\mathrm{SU}(2,2 \mid 4)$ algebra contains $\mathrm{SO}(2,4)$ as a bosonic subalgebra. We begin by discussing the Dirac matrices of $\mathrm{SO}(2,4)$ and $\mathrm{SO}(6)$. We index the $\mathrm{SO}(2,4)$ directions by $\hat{m}=\{m, S, T\}$, with $m=0, \ldots, 3$ and signature $\hat{\eta}_{\hat{m} \hat{n}}=\operatorname{diag}(-++++-)$. Written in a chiral basis, the $\mathrm{SO}(2,4)$ Dirac matrices have the form

$$
\hat{\Gamma}_{m}=\left(\begin{array}{cc}
0 & \gamma_{m} \\
\gamma_{m} & 0
\end{array}\right), \quad \hat{\Gamma}_{S}=\left(\begin{array}{cc}
0 & \gamma_{5} \\
\gamma_{5} & 0
\end{array}\right), \quad \hat{\Gamma}_{T}=\left(\begin{array}{cc}
0 & -1 \\
1 & 0
\end{array}\right),
$$

where the $\gamma_{m}$ are the Dirac matrices of $\mathrm{SO}(1,3)$, and $\gamma_{5}=i \gamma_{0} \gamma_{1} \gamma_{2} \gamma_{3}$.

The chiral decomposition proves useful, since the minimal spinor in $A d S_{5} \times S^{5}$ dimensions is chiral with respect to both $\mathrm{SO}(2,4)$ and $\mathrm{SO}(6)$. We will work with right-handed spinors $\lambda$ satisfying $\hat{\Gamma}_{7} \lambda=-\lambda$, where

$$
\Gamma_{7}=i \hat{\Gamma}_{0} \hat{\Gamma}_{1} \cdots \hat{\Gamma}_{S} \hat{\Gamma}_{T}=\left(\begin{array}{cc}
1 & 0 \\
0 & -1
\end{array}\right)
$$

\footnotetext{
${ }^{6}$ In [i $[\overline{1}$, a different coset representative is used to prove (4.2i) to all orders, and the corrections in $(4.6 i)$ are attributed to Baker-Hausdorff rearrangements.
} 
We denote the restriction of $\hat{\Gamma}$ to the right-handed chiral spinor subspace by $\hat{\gamma}$. The antisymmetrized products of the $\hat{\gamma}_{\hat{m}}$ are given by

$$
\hat{\gamma}_{m n}=\gamma_{m n}, \quad \hat{\gamma}_{m S}=\gamma_{m} \gamma_{5}, \quad \hat{\gamma}_{m T}=-\gamma_{m}, \quad \hat{\gamma}_{T S}=\gamma_{5}
$$

These matrices satisfy the Fierz identities

$$
\left(\hat{\gamma}_{\hat{m} \hat{n}}\right)_{\alpha}{ }^{\beta}\left(\hat{\gamma}^{\hat{m} \hat{n}}\right)_{\gamma}{ }^{\delta}=2 \delta_{\alpha}{ }^{\beta} \delta_{\gamma}{ }^{\delta}-8 \delta_{\alpha}{ }^{\delta} \delta_{\gamma}{ }^{\beta}, \quad\left(\hat{\gamma}_{\hat{m} \hat{n}}\right)_{\alpha}{ }^{\beta}\left(\hat{\gamma}^{\hat{p} \hat{q}}\right)_{\beta}{ }^{\alpha}=-8 \delta_{[\hat{m}}{ }^{\hat{p}} \delta_{\hat{n}]}^{\hat{q}}
$$

The $\mathrm{SO}(6)$ directions are indexed by $I$. We denote the Dirac matrices of $\mathrm{SO}(6)$, restricted to the chiral subspace, by $\hat{\gamma}^{\prime I}$. If we divide the index $I$ as $I=\left\{T^{\prime}, m^{\prime}\right\}$, where $m^{\prime}=1, \ldots, 5$ runs over the $S^{5}$ directions, and consider antisymmetrized

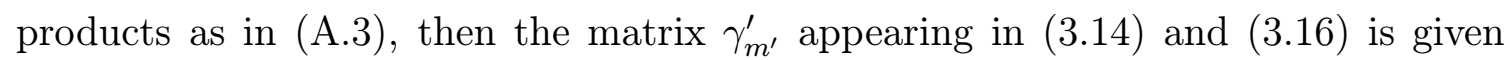
by $\gamma_{m^{\prime}}^{\prime}=\hat{\gamma}_{m^{\prime} T^{\prime}}^{\prime}$.

The group $\mathrm{SU}(2,2 \mid 4)$ is generated by: $\mathrm{SO}(2,4)$ transformations $\hat{M}_{\hat{m} \hat{n}}(\hat{m}, \hat{n}=$ $0, \ldots, 3, S, T)$; SU(4) transformations $U_{i}^{j}(i, j=1, \ldots, 4)$; and fermionic transformations $\mathcal{Q}_{\alpha}^{i}(\alpha=1, \ldots, 4)$ and $\mathcal{Q}_{i}^{\alpha}=i\left(\left(\mathcal{Q}^{i}\right)^{\dagger} \gamma_{0}\right)^{\alpha}$. The structure of the algebra is

$$
\begin{aligned}
{\left[\hat{M}_{\hat{m} \hat{n}}, \hat{M}_{\hat{p} \hat{q}}\right] } & =\hat{\eta}_{\hat{m}[\hat{p}} \hat{M}_{\hat{q}] \hat{n}}-\hat{\eta}_{\hat{n}[\hat{p}} \hat{M}_{\hat{q}] \hat{m}} \\
{\left[\hat{M}_{\hat{m} \hat{n}}, \mathcal{Q}_{\alpha}{ }^{i}\right] } & =-\frac{1}{4}\left(\hat{\gamma}_{\hat{m} \hat{n}} \mathcal{Q}^{i}\right)_{\alpha} \\
\left\{\mathcal{Q}_{\alpha}{ }^{i}, \overline{\mathcal{Q}}_{j}{ }^{\beta}\right\} & =\frac{1}{2} \delta_{j}{ }^{i}\left(\hat{\gamma}^{\hat{m} \hat{n}}\right)_{\alpha}{ }^{\beta} \hat{M}_{\hat{m} \hat{n}}-\delta_{\alpha}{ }^{\beta} U_{j}{ }^{i} \\
{\left[U_{i}^{j}, \mathcal{Q}_{\alpha}^{k}\right] } & =\delta_{i}{ }^{k} \mathcal{Q}_{\alpha}{ }^{j}-\frac{1}{4} \delta_{i}{ }^{j} \mathcal{Q}_{\alpha}{ }^{k} \\
{\left[U_{i}{ }^{j}, U_{k}\right] } & =\delta_{i}{ }^{l} U_{k}{ }^{j}-\delta_{k}{ }^{j} U_{i}{ }^{l}
\end{aligned}
$$

plus complex conjugates. All other commutators vanish.

We can rewrite this in two ways, corresponding to two different breakdowns of the $\mathrm{SO}(2,4)$ subalgebra.

\section{A.1 The AdS decomposition}

The AdS decomposition takes note of the coset structure

$$
A d S_{5}=\frac{\mathrm{SO}(2,4)}{\mathrm{SO}(1,4)}
$$

We split the generators of $\mathrm{SO}(2,4)$ into

$$
\tilde{P}_{\tilde{m}}=2 \hat{M}_{\tilde{m} T}, \quad \tilde{M}_{\tilde{m} \tilde{n}}=\hat{M}_{\tilde{m} \tilde{n}}
$$

with $\tilde{m}, \tilde{n}=0,1,2,3,4=S$. The $\tilde{M}_{\tilde{m} \tilde{n}}$ generate the stability $\operatorname{SO}(1,4)$, while the $\tilde{P}_{\tilde{m}}$ 
generate translations in $A d S_{5}$. In this decomposition, the $\mathrm{SU}(2,2 \mid 4)$ algebra is

$$
\begin{aligned}
{\left[\tilde{M}_{\tilde{m} \tilde{n}}, \tilde{M}_{\tilde{p} \tilde{q}}\right] } & =\tilde{\eta}_{m[\tilde{p}} \tilde{M}_{\tilde{q}] \tilde{n}}-\tilde{\eta}_{\tilde{n}[\tilde{p}} \tilde{M}_{\tilde{q}] \tilde{m}}, \\
{\left[\tilde{P}_{\tilde{q}}, \tilde{M}_{\tilde{m} \tilde{n}}\right] } & =\tilde{\eta}_{\tilde{q}[\tilde{m}} \tilde{P}_{\tilde{n}]}, \quad\left[\tilde{P}_{\tilde{m}}, \tilde{P}_{\tilde{n}}\right]=2 \tilde{M}_{\tilde{m} \tilde{n}}, \\
{\left[\tilde{M}_{\tilde{m} \tilde{n}}, \mathcal{Q}_{\alpha}{ }^{i}\right] } & =-\frac{1}{4}\left(\hat{\gamma}_{\tilde{m} \tilde{n}} \mathcal{Q}^{i}\right)_{\alpha}, \\
{\left[\tilde{P}_{\tilde{m}}, \mathcal{Q}_{\alpha}{ }^{i}\right] } & =\frac{1}{2}\left(\hat{\gamma}_{\tilde{m} T} \mathcal{Q}^{i}\right)_{\alpha}, \\
\left\{\mathcal{Q}_{\alpha}{ }^{i}, \overline{\mathcal{Q}}_{j}{ }^{\beta}\right\} & =\frac{1}{2} \delta_{j}{ }^{i}\left(\hat{\gamma}^{\tilde{m} T}\right)_{\alpha}{ }^{\beta} \tilde{P}_{\tilde{m}}+\frac{1}{2} \delta_{j}{ }^{i}\left(\hat{\gamma}^{\tilde{m} \tilde{n}}\right)_{\alpha}{ }^{\beta} \tilde{M}_{\tilde{m} \tilde{n}}-\delta_{\alpha}{ }^{\beta} U_{j}{ }^{i}, \\
{\left[U_{i}{ }^{j}, \mathcal{Q}_{\alpha}^{k}\right] } & =\delta_{i}{ }^{k} \mathcal{Q}_{\alpha}{ }^{j}-\frac{1}{4} \delta_{i}{ }^{j} \mathcal{Q}_{\alpha}{ }^{k}, \\
{\left[U_{i}{ }^{j}, U_{k}{ }^{l}\right] } & =\delta_{i}{ }^{l} U_{k}{ }^{j}-\delta_{k}{ }^{j} U_{i}{ }^{l},
\end{aligned}
$$

plus complex conjugates. All other commutators vanish.

\section{A.2 The superconformal decomposition}

In the superconformal decomposition of $\mathrm{SU}(2,2 \mid 4)$, the $\mathrm{SO}(2,4)$ subalgebra is decomposed by weight under the dilatation operator

$$
D=2 \hat{M}_{T S}
$$

The four-dimensional translations

$$
P_{m}=2\left(\hat{M}_{m T}+\hat{M}_{m S}\right)
$$

$(m=0,1,2,3)$ have weight 1 (i.e. $\left.\left[D, P_{m}\right]=P_{m}\right)$; the $\mathrm{SO}(1,3)$ Lorentz transformations

$$
M_{m n}=\hat{M}_{m n}
$$

the SU(4) transformations $U_{i}^{j}$ and the dilatation operator $D$ itself have weight zero; and the special conformal transformations

$$
K_{m}=2\left(\hat{M}_{m T}-\hat{M}_{m S}\right)
$$

have weight -1 . The fermionic operators split into supersymmetries $Q$, with weight $1 / 2$, and special supersymmetries $S$, with weight $-1 / 2$, according to

$$
Q_{\alpha}^{i}=\frac{1}{\sqrt{2}}\left(1-\gamma_{5}\right)_{\alpha}{ }^{\beta} \mathcal{Q}_{\beta}{ }^{i}, \quad S_{\alpha}^{i}=\frac{1}{\sqrt{2}}\left(1+\gamma_{5}\right)_{\alpha}{ }^{\beta} \mathcal{Q}_{\beta}{ }^{i}
$$

That is, $D$ divides the fermionic generators according to their $\mathrm{SO}(1,3)$ chirality. In 
this decomposition, the algebra becomes

$$
\begin{aligned}
{\left[M_{m n}, M_{p q}\right] } & =\eta_{m[p} M_{q] n}-\eta_{n[p} M_{q] m}, \\
{\left[P_{q}, M_{m n}\right] } & =\eta_{q[m} P_{n]}, \quad\left[K_{q}, M_{m n}\right]=\eta_{q[m} K_{n]}, \\
{\left[D, P_{m}\right] } & =P_{m}, \quad\left[D, K_{m}\right]=-K_{m}, \\
{\left[P_{m}, K_{n}\right] } & =2\left(\eta_{m n} D+2 M_{m n}\right), \\
{\left[M_{m n}, Q_{\alpha}{ }^{i}\right] } & =-\frac{1}{4}\left(\gamma_{m n} Q^{i}\right)_{\alpha}, \quad\left[M_{m n}, S_{\alpha}{ }^{i}\right]=-\frac{1}{4}\left(\gamma_{m n} S^{i}\right)_{\alpha}, \\
{\left[P_{m}, S_{\alpha}{ }^{i}\right] } & =\left(\gamma_{m} Q^{i}\right)_{\alpha}, \quad\left[K_{m}, Q_{\alpha}{ }^{i}\right]=\left(\gamma_{m} S^{i}\right)_{\alpha}, \\
{\left[D, Q_{\alpha}{ }^{i}\right] } & =\frac{1}{2} Q_{\alpha}^{i}, \quad\left[D, S_{\alpha}{ }^{i}\right]=-\frac{1}{2} S_{\alpha}^{i}, \\
\left\{Q_{\alpha}{ }^{i}, \bar{Q}_{j}{ }^{\beta}\right\} & =\delta_{j}{ }^{i}\left(\gamma^{m}\right)_{\alpha}{ }^{\beta} P_{m}, \quad\left\{S_{\alpha}{ }^{i}, \bar{S}_{j}{ }^{\beta}\right\}=\delta_{j}{ }^{i}\left(\gamma^{m}\right)_{\alpha}{ }^{\beta} K_{m}, \\
\left\{Q_{\alpha}{ }^{i}, \bar{S}_{j}{ }^{\beta}\right\} & =\delta_{j}{ }^{i} \delta_{\alpha}{ }^{\beta} D+\delta_{j}{ }^{i}\left(\gamma^{m n}\right)_{\alpha}{ }^{\beta} M_{m n}-2 \delta_{\alpha}{ }^{\beta} U_{j}{ }^{i}, \\
{\left[U_{i}{ }^{j}, Q_{\alpha}^{k}\right] } & =\delta_{i}{ }^{k} Q_{\alpha}{ }^{j}-\frac{1}{4} \delta_{i}{ }^{j} Q_{\alpha}{ }^{k}, \\
{\left[U_{i}{ }^{j}, S_{\alpha}^{k}\right] } & =\delta_{i}{ }^{k} S_{\alpha}{ }^{j}-\frac{1}{4} \delta_{i}{ }^{j} S_{\alpha}{ }^{k}, \\
{\left[U_{i}{ }^{j}, U_{k}{ }^{l}\right] } & =\delta_{i}{ }^{l} U_{k}{ }^{j}-\delta_{k}{ }^{j} U_{i}{ }^{l},
\end{aligned}
$$

plus complex conjugates. All other commutators vanish.

\section{A.3 Fermionic coordinates of $\mathrm{SU}(2,2 \mid 4)$ cosets}

A general minimal spinor in $A d S_{5} \times S^{5}$ is chiral with respect to $\mathrm{SO}(2,4)$ and $\mathrm{SO}(6)$, and so contains 32 degrees of freedom. We write the 32 fermionic coordinates of the $A d S_{5} \times S^{5}$ superspace $C_{(10 \mid 32)}$ as $\Theta_{\alpha}^{i}, \bar{\Theta}_{i}^{\alpha}$, where $\alpha=1,2,3,4$ and $i=1,2,3,4$ are the $\mathrm{SO}(2,4)$ and $\mathrm{SO}(6)$ spinor indices. As in the discussion above, the conjugate spinor $\bar{\Theta}_{i}^{\alpha}$ is defined by

$$
\bar{\Theta}_{i}^{\alpha}=i\left(\left(\Theta^{i}\right)^{\dagger} \gamma_{0}\right)^{\alpha}
$$

The superconformal decomposition splits the fermionic generators with respect to their $\mathrm{SO}(1,3)$ chirality, which brings about a split of the coordinate $\Theta_{\alpha}^{i}$ into

$$
\begin{aligned}
\theta_{\alpha}^{i} & =\frac{1}{2 \sqrt{2}}\left(\left(1+\gamma_{5}\right) \Theta^{i}\right)_{\alpha}, \\
\vartheta_{\alpha}^{i} & =\frac{1}{2 \sqrt{2}}\left(\left(1-\gamma_{5}\right) \Theta^{i}\right)_{\alpha} .
\end{aligned}
$$

The $\theta_{\alpha}^{i}$ are the coordinates conjugate to the supersymmetries $Q$, and the $\vartheta_{a}^{i}$ are conjugate to the special supersymmetries $S$.

In the conformal superspaces $C_{(4 \mid 16)}$ and $C_{(10 \mid 16)}$, the $Q_{\alpha}^{i}$ are elements of the coset and have conjugate coordinates $\theta_{\alpha}^{i}$, but the $S_{\alpha}^{i}$ are elements of the stability group and have no corresponding conjugate coordinates. 


\section{B. The superisometries of $A d S_{5} \times S^{5}$ to quartic order in fermions}

We present to quartic order in the fermions the bosonic vielbein $E^{m}$ of $C_{(10 \mid 32)}$ and the coordinate variations under supersymmetries and special supersymmetries, in the limit $\rho \rightarrow \infty$. These are needed to establish the coordinate transformations $\left(\bar{A}_{.} \bar{G}_{1}^{\prime}\right)-\left(\bar{A}_{-} \bar{Z}_{1}\right)$ and the corresponding matching of superisometries and superconformal transformations. We have explicitly calculated the matching only for the supersymmetries and special supersymmetries, but we expect the matching to hold for all superisometries.

$$
\begin{aligned}
E^{m}=\rho\left[d x^{n} \delta_{n}{ }^{m}+\frac{1}{2}(\right. & \left(d \bar{\theta}_{i}+\bar{\vartheta}_{i} \gamma_{n} d x^{n}\right) \gamma^{m} \theta^{i}+\frac{1}{4}\left(\bar{\theta}_{i} d \vartheta^{j}\right)\left(\bar{\theta}_{j} \gamma^{m} \theta^{i}\right)+ \\
& +\frac{1}{4} \bar{\vartheta}_{j}\left(d \theta^{i}-d x^{n} \gamma_{n} \vartheta^{i}\right)\left(\bar{\theta}_{i} \gamma^{m} \theta^{j}\right)+ \\
& \left.\left.+\frac{1}{12}\left(\bar{\theta}_{j} \gamma^{m}\left(d \theta^{i}-d x^{n} \gamma_{n} \vartheta^{i}\right)\right)\left(\bar{\theta}_{i} \vartheta^{j}+\bar{\vartheta}_{i} \theta^{j}\right)+\text { h.c. }\right)\right], \\
\delta x^{m}=- & \frac{1}{4}\left(\bar{\epsilon}_{j} \vartheta^{i} \bar{\theta}_{i} \gamma^{m} \theta^{j}+\bar{\eta}_{j} \theta^{i} \bar{\theta}_{i} \gamma^{m} \theta^{j}-\bar{\vartheta}_{i} \epsilon^{j} \bar{\theta}_{j} \gamma^{m} \theta^{i}-\bar{\theta}_{i} \eta^{j} \bar{\theta}_{j} \gamma^{m} \theta^{i}\right)- \\
& -\frac{1}{12}\left(\bar{\epsilon}_{j} \gamma^{m} \theta^{i} \bar{\theta}_{i} \vartheta^{j}+\bar{\epsilon}_{j} \gamma^{m} \theta^{i} \bar{\vartheta}_{i} \theta^{j}-\bar{\theta}_{i} \gamma^{m} \epsilon^{j} \bar{\vartheta}_{j} \theta^{i}-\bar{\theta}_{i} \gamma^{m} \epsilon^{j} \bar{\theta}_{j} \vartheta^{i}\right), \\
\delta \theta^{i}=- & \epsilon^{i}-\frac{4}{3}\left(\bar{\epsilon}_{j} \vartheta^{i}+\bar{\eta}_{j} \theta^{i}\right) \theta^{j}+\frac{2}{3}\left(\epsilon^{j}\left(\bar{\theta}_{j} \vartheta^{i}+\bar{\vartheta}_{j} \theta^{i}\right)+\left(\bar{\theta}_{j} \eta^{i}+\bar{\vartheta}_{j} \epsilon^{i}\right) \theta^{j}\right)- \\
& -\frac{1}{2}\left(\bar{\epsilon}_{j} \gamma^{a} \theta^{j}-\bar{\theta}_{j} \gamma^{a} \epsilon^{j}\right)\left(\gamma_{a} \vartheta\right)^{i}, \\
\delta y^{I}= & \frac{1}{2}\left[\bar{\epsilon}_{i} \vartheta^{j}\left(\delta^{I J} \mathbb{1}-\hat{\gamma}^{I J}\right)_{j}{ }^{i}+\bar{\vartheta}_{i} \epsilon^{j}\left(\delta^{I J} \mathbb{1}+\hat{\gamma}^{I J}\right)_{j}{ }^{i}-\right. \\
& \left.-\bar{\eta}_{i} \theta^{j}\left(\delta^{I J} \mathbb{1}+\hat{\gamma}^{I J}\right)_{j}{ }^{i}-\bar{\theta}_{i} \eta^{j}\left(\delta^{I J} \mathbb{1}-\hat{\gamma}^{I J}\right)_{j}{ }^{i}\right] y^{J} .
\end{aligned}
$$

\section{References}

[1] J. Maldacena, The large- $N$ limit of superconformal field theories and supergravity,

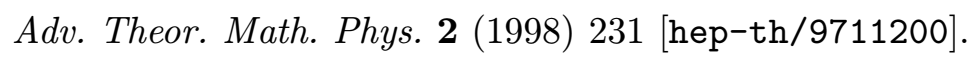

[2] L. Castellani, R. D'Auria and P. Fré, Supergravity and superstrings: a geometric perspective, Vols. 1,2,3, World Scientific, Singapore 1991.

[3] P. Claus and R. Kallosh, Superisometries of the AdS $\times S$ superspace, 'U.

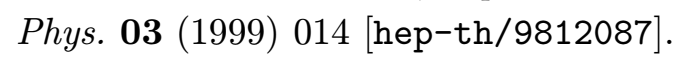

[4] D. Sorokin, Superbranes and superembeddings, 'Phhys. [hep-th/9906142].

[5] P.S. Howe and E. Sezgin, Superbranes, iPhys. Lett. [hep-th/96072 $2 \overline{7}$ in. 
[6] J. Maldacena, Wilson loops in large-N field theories, 'Phys. Rev. Lett. 80 (1998) [hep-th/9803002in.

[7] S.-J. Rey and J. Yee, Macroscopic strings as heavy quarks in large- $N$ gauge theory and anti-de Sitter supergravity, hep-th/9803001:.

[8] E. Witten, Anti-de Sitter space, thermal phase transition and confinement in gauge theories, 'Ádv. Theor. Math. Phys. 2 (1998) 505i [hep-th/9803131].

[9] S. Forste, D. Ghoshal and S. Theisen, Stringy corrections to the Wilson loop in $N=4$

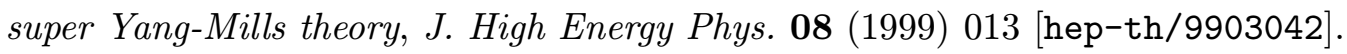

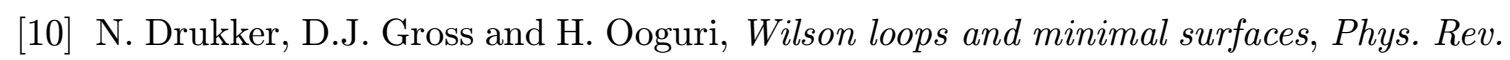
i . D 60 (1999) 125006 [hep-th/9904191i].

[11] H. Ooguri, J. Rahmfeld, H. Robins, J. Tannenhauser, Holography in superspace, in preparation.

[12] H. Liu and A.A. Tseytlin, $D=4$ super Yang-Mills, $D=5$ gauged supergravity and

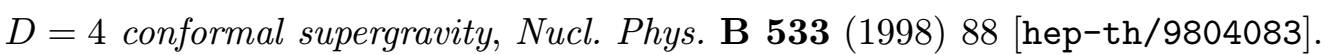

[13] M. Nishimura and Y. Tanii, Local symmetries in the $A d S_{7} / C F T_{6}$ correspondence, Mod. Phys. Lett. A 14 (1999) 2709: [hep-th/9910192].

[14] V. Balasubramanian, E. Gimon, D. Minic and J. Rahmfeld, 4d conformal supergravity from $A d S$ space, in preparation.

[15] M. Nishimura and Y. Tanii, Supersymmetry in the AdS/CFT correspondence, 'P

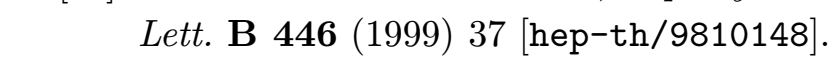

[16] M. Nishimura, Conformal supergravity from the AdS/CFT correspondence, hep-th/0004179.'.

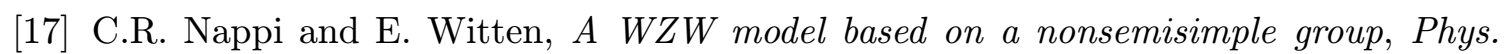
'- Revt

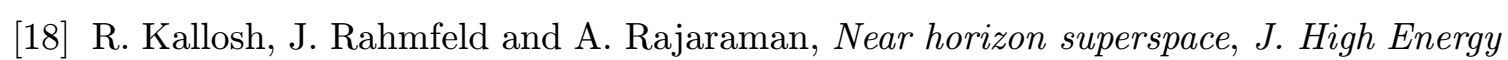
:

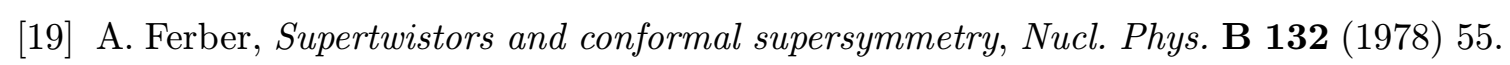

[20] H. Lu, C.N. Pope and J. Rahmfeld, A construction of killing spinors on $S^{n}$, : 